\title{
Proposal of Primary Endpoints for TACE Combination Trials with Systemic Therapy: Lessons Learned from 5 Negative Trials and the Positive TACTICS Trial
}

\author{
Masatoshi Kudo \\ Department of Gastroenterology and Hepatology, Kindai University Faculty of \\ Medicine, Osaka-Sayama, Japan
}

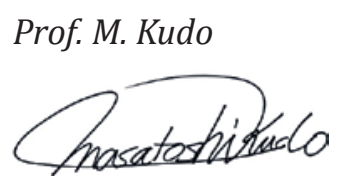

Editor Liver Cancer

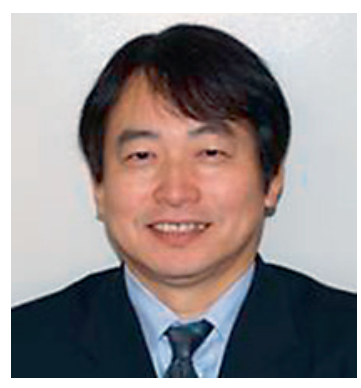

The multikinase inhibitor sorafenib is the first oral molecular targeted agent to improve survival in patients with unresectable advanced hepatocellular carcinoma (HCC). Various clinical trials have been conducted with the ultimate goal of extending survival not only in advanced HCC patients, but also in intermediate-stage HCC patients. The aim of these trials was to test the hypothesis that combination therapy with transcatheter arterial chemoembolization (TACE) and molecular targeted agents (1) attenuates the release of vascular endothelial growth factor and other angiogenic growth factors in response to hypoxia induced by TACE and (2) prevents a decrease in hepatic functional reserve by extending the interval between TACE sessions through suppression of residual tumor proliferation. Five trials investigating combination therapy with TACE and molecular targeted agents have ended in failure. In the wake of these failures, the recent positive TACTICS trial, which combined TACE and sorafenib and reported extension of the primary endpoint of progression-free survival, was a breakthrough. This editorial interprets the design of the TACTICS trial, provides an in-depth review of factors related to its success by comparison with previous failed trials, and discusses the lessons learned regarding the endpoints to be considered in future trials of combination therapy with TACE. 


\section{Liver Cancer}

\section{Introduction}

The efficacy of the molecular targeted agent sorafenib in patients with unresectable advanced hepatocellular carcinoma (HCC) was demonstrated in the SHARP trial [1] and in a trial conducted in the Asia-Pacific region [2]. Sorafenib is considered a first-line drug for patients with unresectable advanced HCC. The first-line treatment for patients with intermediate-stage HCC is transcatheter arterial chemoembolization (TACE); however, the release of large concentrations of angiogenic factors, such as vascular endothelial growth factor (VEGF), triggered by hypoxia induced by TACE results in tumor progression and recurrence [3-5]. Another disadvantage of TACE is that repeated procedures reduce liver function [6]; therefore, minimizing the number of TACE sessions is a critical challenge in the treatment of intermediate-stage HCC patients. Several clinical trials have attempted to address this problem by combining TACE with molecular targeted agents; however, to date, all have ended in failure [7-11], and the combination of TACE and molecular targeted agents is therefore not recommended in routine practice.

In a subanalysis within the SHARP trial, the hazard ratio (HR) for overall survival (OS) in potential TACE candidates (no vascular invasion or extrahepatic spread) was a very favorable 0.52 , clearly demonstrating that sorafenib extended the median OS by a factor of approximately 1.5 [12]. These results suggest that the use of sorafenib in adjuvant, combination, or sequential therapy may extend survival in TACE candidates. The effects of combining sorafenib and TACE go beyond the combination of two different treatment modalities. Sorafenib shows promise for inhibiting recurrence and repeated tumor growth after TACE because it attenuates the acceleration of angiogenesis after the procedure, suggesting it can extend the period during which tumor progression is controllable by TACE. In addition, it may help prevent worsening of hepatic function by reducing the number of TACE sessions required. In summary, sorafenib shows promise for improving survival by extending time to progression (TTP) to advanced-stage HCC in patients with intermediate-stage HCC that are eligible for TACE.

\section{Overview of the TACTICS Trial}

\section{Trial Design}

The TACTICS trial was a multicenter prospective randomized controlled trial comparing TACE plus sorafenib with TACE alone that was conducted at 33 sites in Japan (Fig. 1). A total of 156 patients with unresectable HCC were assigned to receive sorafenib plus TACE $(n=80)$ or TACE alone $(n=76)$ at a 1:1 ratio. The inclusion criteria were Child-Pugh score $\leq 7$, a maximum of two previous TACE sessions, and $\leq 10 \mathrm{HCCs}$ with none exceeding $10 \mathrm{~cm}$ in size. The exclusion criteria were extrahepatic spread and vascular invasion. Patients in the TACE plus sorafenib arm started sorafenib 2-3 weeks before TACE at a dose of $400 \mathrm{mg}$ once daily. The purpose of this sequential pretreatment with sorafenib was to assess tolerability to sorafenib, normalize the tumor vasculature to improve TACE effectiveness, and attenuate VEGF upregulation after the TACE procedure. Sorafenib was temporarily suspended 2 days before and after TACE. In patients showing sorafenib tolerance, the dose was increased to 800 mg daily when possible. TACE was performed on demand, and repeated TACE was generally performed in cases with viable lesions that grew by $\geq 50 \%$ over baseline. Response was assessed using computed tomography, magnetic resonance imaging, or other related modalities every 8 weeks. The study had two co-primary endpoints, namely, progression-free survival (PFS) and OS, and adopted a gatekeeping strategy. The secondary endpoints were the time until TACE was no longer feasible or no longer showed any benefit (time to unTACEable progression: TTUP), TTP, response rate, and safety. As further explained below, the 


\section{Trial design of TACTICS}

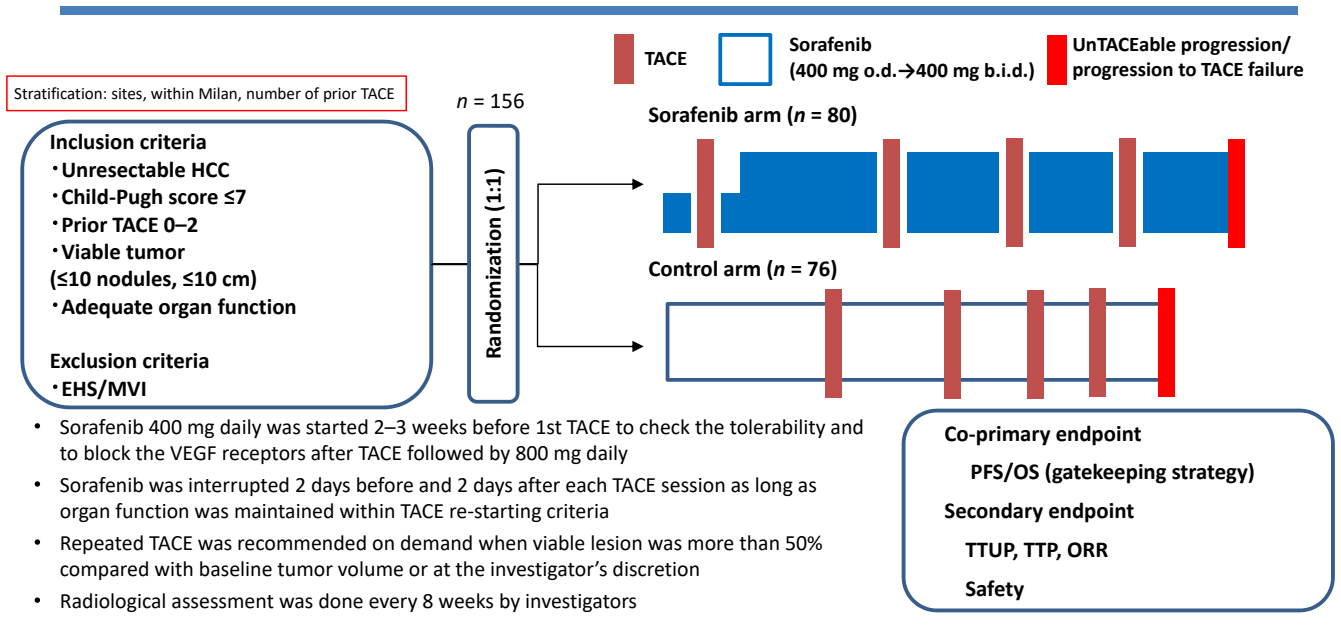

Fig. 1. Trial design of TACTICS.

development of new intrahepatic lesions was not defined as tumor progression. This criterion was introduced to maximize the duration of sorafenib administration and to keep the progression criteria for TACE as consistent as possible with those currently used in clinical practice. Use of the RECIST criteria as response evaluation criteria/a stopping rule is inappropriate because repeated TACE is generally performed after detecting a new intrahepatic lesion, which does not qualify as treatment failure requiring a switch to a nextline of treatment. Therefore, the TACE progression criteria were created specifically for the TACTICS trial and were consistent with those used in clinical practice.

The criteria for progression with TACE (unTACEable progression) were (1) $\geq 25 \%$ increase in intrahepatic viable lesions, (2) decline in hepatic functional reserve to Child-Pugh class C, (3) appearance of extrahepatic lesions, (4) appearance of vascular invasion, or (5) meeting the Japan Society of Hepatology criteria for TACE-refractory disease [13]. Therefore, PFS was defined as the time to either unTACEable progression or death. The most important feature of the TACTICS trial design is that the RECIST criteria were not used, and consequently the development of new intrahepatic lesions was not considered progression. This enabled long-term administration of sorafenib.

\section{Results of the TACTICS Trial}

The results for the primary endpoint of PFS were very favorable, with a median of 25.2 months in the TACE plus sorafenib arm and 13.5 months in the TACE alone arm (HR, 0.59; $p=0.006$; Table 1) [14]. TTUP results were also favorable, with a median of 26.7 months in the TACE plus sorafenib arm and 20.6 months in the TACE alone arm (HR, 0.57; $p=0.02$; Table 1). Similarly, TTP results were favorable, with a median of 26.7 months in the TACE plus sorafenib arm and 16.4 months in the TACE alone arm (HR, 0.54; $p=0.005)$. PFS results were also better for the TACE plus sorafenib arm in all subgroup analyses. The response rates after the first TACE session did not differ significantly between the arms. There were no unexpected adverse events. The median duration of sorafenib administration was long at 38.7 months, and the median daily dose was somewhat low at $355.2 \mathrm{mg}$. The interval between TACE sessions was 21.1 weeks in the TACE plus sorafenib arm, which was significantly longer 
Table 1. Results of TACTICS trial

\begin{tabular}{lllll}
\hline & TACE with sorafenib & TACE alone & HR $(95 \%$ CI $)$ & $p$ value \\
\hline PFS & 25.2 & 13.5 & $0.59(0.41-0.87)$ & 0.006 \\
TTUP & 26.7 & 20.6 & $0.57(0.36-0.92)$ & 0.02 \\
TTP & 26.7 & 16.4 & $0.54(0.35-0.83)$ & 0.005 \\
TTVI & 31.3 & 4.0 & $0.26(0.09-0.75)$ & 0.005 \\
TTEHS & 15.7 & 6.9 & $0.21(0.06-0.70)$ & 0.006 \\
TTSP & 22.5 & 6.3 & $0.31(0.15-0.63)$ & 0.001 \\
\hline
\end{tabular}

Data are presented as median values in months. TACE, transcatheter arterial chemoembolization; HR, hazard ratio; PFS, progression-free survival; TTUP, time to unTACEable progression; TTP, time to progression; TTVI, time to vascular invasion; TTEHS, time to extrahepatic spread; TTSP, time to stage progression.

than the interval of 16.9 weeks in the TACE alone arm $(p=0.018)$. Other parameters that were significantly longer in the TACE plus sorafenib arm than in the TACE alone arm were time to detection of vascular invasion (31.3 vs. 4.0 months), time to detection of extrahepatic spread (15.7 vs. 6.9 months), and time to stage progression (22.5 vs. 6.3 months) (Table 1).

\section{Reasons for the Success of the TACTICS Trial}

The TACTICS trial, which demonstrated that TACE plus sorafenib extended the primary endpoint of PFS compared with TACE alone, was the world's first positive trial of combination therapy with TACE and a molecular targeted agent. The results of combination therapy were also superior to those of TACE alone for all secondary endpoints except response rate. The key contributing factor to the positive results was the long duration of sorafenib administration (38.7 weeks). The reasons for this long administration period were that (1) the development of new intrahepatic lesions was not considered progression, and (2) new progression criteria optimized for TACE (unTACEable progression) were developed, and TACE-specific PFS was defined according to those criteria.

\section{Reasons for the Failure of Past Negative Trials}

\section{Post-TACE Trial}

Background

Although TACE is performed as first-line treatment for unresectable HCC, it is rarely curative. Repeated TACE is common; however, this strategy can worsen hepatic function. Post-TACE recurrence is believed to be caused by factors such as increased angiogenesis and high VEGF expression. The trial was designed to test the hypothesis that administration of sorafenib, a tyrosine kinase inhibitor that targets Raf, VEGF receptor, platelet-derived growth factor receptor, and other factors involved in tumor cell proliferation and angiogenesis would delay post-TACE recurrence and consequently extend survival time. The Post-TACE trial was a Phase III placebo-controlled trial conducted in Japan and South Korea [7].

Trial Design

The subjects were patients with unresectable HCC and Child-Pugh A hepatic functional reserve who had responded to TACE as demonstrated by imaging assessment after 1-3 months. Responders were assigned to sorafenib and placebo arms. The primary endpoint was TTP and the secondary endpoint was OS. 


\section{Liver
Cancer}

\begin{tabular}{l|l}
\hline Liver Cancer 2018;7:225-234 \\
\hline DOI: 10.1159/000492535 & $\begin{array}{l}\text { @ 2018 S. Karger AG, Basel } \\
\text { www.karger.com/lic }\end{array}$ \\
\hline
\end{tabular}

Kudo: Proposal of Endpoints for TACE Combination Trials with Systemic Therapy

Table 2. TACE combination trials with sorafenib

\begin{tabular}{|c|c|c|c|c|}
\hline Trial & Ph3 Post-TACE & Ph2 SPACE & Ph3 TACE-2 & $\mathrm{Ph} 2$ TACTICS \\
\hline Author & Kudo et al. [7], 2011 & Lencioni et al. [9], 2016 & $\begin{array}{l}\text { Meyer et al. [11], } \\
2017\end{array}$ & $\begin{array}{l}\text { Kudo M et al. [14], } \\
2018\end{array}$ \\
\hline Child-Pugh class & A & A (no ascites) & A & A5-B7 \\
\hline ECOG-PS & $0-1$ & 0 & $0-1$ & $0-1$ \\
\hline Tumor burden & $\begin{array}{l}\leq 7 \mathrm{~cm} \\
\leq 10 \text { tumors }\end{array}$ & $\begin{array}{l}\text { Unresectable } \\
\text { multinodular }\end{array}$ & $\begin{array}{l}\text { Not a candidate for } \\
\text { resection or } \\
\text { transplantation }\end{array}$ & $\begin{array}{l}\leq 10 \mathrm{~cm} \\
\leq 10 \text { tumors }\end{array}$ \\
\hline TACE procedure & cTACE, on demand & DEB-TACE, scheduled & DEB-TACE, on demand & cTACE, on demand \\
\hline Endpoint & TTP (5.4 months) & TTP (5.6 months) & PFS (8.5 months) & PFS (25.2 months) \\
\hline Progression criteria & RECICL 2004 & mRECIST & RECIST 1.1 & $\begin{array}{l}\text { UnTACEable } \\
\text { progression/TACE } \\
\text { failure New lesion: } \\
\text { not PD }\end{array}$ \\
\hline Sorafenib duration, weeks & 17.0 & 21.0 & 17.1 & 38.7 \\
\hline $\begin{array}{l}\text { Median follow-up, } \\
\text { weeks }\end{array}$ & NA & 38.6 & 88.6 & 122.3 \\
\hline
\end{tabular}

cTACE, conventional lipiodol TACE; RECICL, response evaluation criteria in the cancer of liver

Results

The trial enrolled 458 patients (387 from Japan and 71 from South Korea) between April 2006 and July 2009. The primary endpoint of median TTP was 5.4 months in the sorafenib arm and 3.7 months in the placebo arm (HR, 0.87; 95\% CI: 0.70-1.09; $p=0.252$; Table 1). The secondary endpoint of median OS was 29.7 months in the sorafenib arm, but the median value was not reached in the placebo arm (HR, 1.06; 95\% CI: $0.69-1.64 ; p=0.790)$. In subgroup analysis, the median TTP in Japanese patients was 3.9 months in the sorafenib arm and 3.7 months in the placebo arm, with a HR of 0.94 and no difference between the arms. In Korean patients, however, the HR was 0.38 , clearly demonstrating a longer TTP in the sorafenib arm.

Interpretation of the Reasons for Failure

In this trial, the primary endpoint of TTP did not significantly differ between the arms. One reason for this could be the timing of sorafenib administration. Because TACE triggers an increase in VEGF production by inducing ischemic conditions, it may be necessary to inhibit angiogenesis soon after TACE to detect the effect of sorafenib. However, the median delay until administration of sorafenib was 9 weeks because the population included only patients who responded to TACE. This delay may be one reason why sorafenib did not have an additive effect.

Another factor contributing to the trial failure was that although TTP did not differ between arms in Japan, the results of sorafenib treatment were good in South Korea. The longer median treatment period in Korean patients than in Japanese patients (31 vs. 16 weeks) was identified as a possible reason for the significant extension of TTP in Korean patients. Therefore, the short sorafenib administration period of 17 weeks was the main reason for the failure of this trial (Table 2). 


\section{Liver

\begin{tabular}{l|l}
\hline Liver Cancer 2018;7:225-234 \\
\hline DOI: 10.1159/000492535 & $\begin{array}{l}\text { ○ 2018 S. Karger AG, Basel } \\
\text { www.karger.com/lic }\end{array}$ \\
\hline
\end{tabular}

Kudo: Proposal of Endpoints for TACE Combination Trials with Systemic Therapy

\section{SPACE Trial}

Background

The SPACE trial was conducted at 85 sites in 13 countries not including Japan. It was a Phase II trial that assessed the safety and efficacy of DEB-TACE with doxorubicin-eluting beads (DEBDOX) plus sorafenib in patients with Barcelona Clinic Liver Cancer (BCLC) stage $B$ unresectable HCC. The trial design incorporated lessons learned from the Post-TACE trial, namely, that sorafenib should be started early to address the increase in VEGF production induced by ischemia after TACE by performing TACE soon after starting sorafenib and then continuing sorafenib after TACE [9].

Trial Design

Patients enrolled at 85 sites in 13 countries were randomly assigned to sorafenib and placebo arms. Sorafenib or placebo was administered 3-7 days before DEB-TACE. Subsequent sessions of DEB-TACE were performed after 3, 7, and 13 months and every 6 months thereafter. The primary endpoint was TTP, and the secondary endpoints were OS, time to extrahepatic spread and vascular invasion, TTUP, and safety. The criteria for unTACEable progression were detection of vascular invasion or extrahepatic spread, persistent ascites, Child-Pugh class B, Eastern Cooperative Oncology Group performance status $\geq 2$, and platelet count $<60,000 / \mu \mathrm{L}$. One-sided significance tests were used for between-arm comparisons with a significance level of $15 \%(\alpha=0.15)$.

Results

Of the 307 patients enrolled, 154 were assigned to the sorafenib arm and 153 to the placebo arm. The primary endpoint of median TTP was 169 days in the sorafenib arm and 166 days in the placebo arm (HR, 0.797; 95\% CI: 0.588-1.08; $p=0.072)$. Although this trial met the statistical significance requirements for a Phase II trial, the results cannot be considered a clinically significant improvement of TTP. The secondary endpoint of OS did not reach the median value in the sorafenib or placebo arm (HR, 0.898; 95\% CI: 0.606-1.33; $p=$ $0.295)$. In addition, time to extrahepatic spread and vascular invasion did not reach median values in the sorafenib or placebo arm (HR, 0.621; 95\% CI: $0.321-1.20 ; p=0.076)$. In the sorafenib and placebo arms, median TTUP was 95 and 224 days, respectively (HR, 1.586; 95\% CI: $1.200-2.096 ; p=0.999$ ), the number of patients with unTACEable progression was 110 and 96 , respectively, and the percentage of patients who only underwent one session of DEB-TACE was 35.9 and $19.2 \%$, respectively.

Comparative analysis between Asian and non-Asian patients showed that the median duration of sorafenib administration was 30 weeks in Asian patients and 17.4 weeks in nonAsian patients. As a result, the HRs for TTP and OS were worse in non-Asian patients $(0.865$ and 1.062, respectively) than in Asian patients (0.720 and 0.677, respectively).

Interpretation of the Reasons for Failure

The primary endpoint of TTP was met to the extent necessary for a Phase II trial, although the results were not clinically significant. There was no difference in the secondary endpoint of OS. Moreover, there was no difference in TTUP, which was shorter in the sorafenib arm the opposite of what was predicted.

The TACE method used in this trial was "scheduled TACE" in which DEB-TACE is performed at 1, 3, 7, and 13 months and every 6 months thereafter. Scheduled TACE involves performing TACE at regular intervals, even if intrahepatic lesions respond to TACE. This approach, which could have reduced hepatic function or increased adverse reactions to sorafenib, is different from the Japanese or Asian approach to treatment of "on-demand" TACE performed when necessary. 


\section{Liver
Cancer}

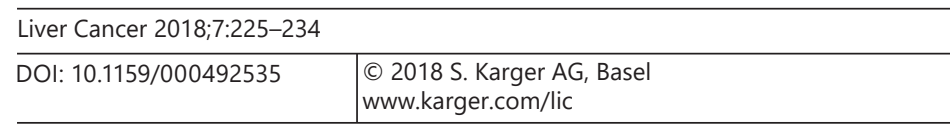

Kudo: Proposal of Endpoints for TACE Combination Trials with Systemic Therapy

Many subjects in this trial underwent few TACE sessions, which may be attributable to inappropriate criteria for unTACEable progression. Although factors such as vascular invasion and extrahepatic spread are appropriate criteria for the discontinuation of TACE, other factors such as Child-Pugh B hepatic functional reserve, persistent ascites, and platelet count $<60,000 / \mu \mathrm{L}$ do not indicate that TACE is unfeasible. The authors of the Post-TACE trial speculated that long-term administration of sorafenib may improve TTP, and these criteria for discontinuation of TACE may have forced the early termination of sorafenib administration in the SPACE trial. As in the Post-TACE trial, the cumulative duration of sorafenib administration in this trial was short at 21 weeks. Considering that TTP and OS were favorable in Asian patients receiving long-term sorafenib treatment (30 weeks) compared with non-Asian patients (17.4 weeks), the short duration of sorafenib administration can be considered the biggest reason for the failure of this trial. In fact, Lencioni et al. [9] in their SPACE Trial paper state "The duration of sorafenib albeit in combination with TACE may be critical for improved outcome."

\section{TACE-2 Trial}

Background

The TACE-2 trial, which was conducted at 20 sites in England, was designed to test the hypothesis that combination treatment with sorafenib and DEB-TACE would inhibit tumor progression and extend OS [10].

Trial Design

Enrolled patients were assigned to sorafenib or placebo arm, and DEB-TACE was performed within 2-5 weeks of sorafenib administration. After that, on-demand DEB-TACE was performed if additional TACE was deemed necessary based on imaging assessment. The primary endpoint was PFS, and the secondary endpoint was OS.

\section{Results}

The 294 enrolled patients were split into two arms, each comprising 147 patients. The primary endpoint of median PFS was 7.8 months in the sorafenib arm and 7.7 months in the placebo arm (HR, 1.03; 95\% CI: 0.75-1.42; $p=0.85$ ). The secondary endpoint of median OS was 18.8 months in the sorafenib arm and 19.6 months in the placebo arm (HR, 1.03; 95\% CI: $0.72-1.49 ; p=0.87$ ).

Interpretation of the Reasons for Failure

In this trial, there was no difference in PFS or OS, indicating that sorafenib was not effective. The criteria for performing repeated TACE were left to the discretion of individual physicians, and repeated TACE before progressive disease (PD) was not prohibited in both arms. Therefore, some patients underwent repeated TACE before sorafenib had the anticipated effect, which indicates that the results were attributable solely to the effects of TACE. This may account for the lack of differences between the arms.

Previous trials reported an additive effect of molecular targeted agents despite the lack of difference between patients receiving TACE plus a molecular targeted agent and those treated with TACE alone. This trial, however, did not show a difference or an additive effect between arms. The STORM trial, which investigated sorafenib adjuvant to locoregional therapy such as resection or radiofrequency ablation, also failed to show the effect of sorafenib [15]. This suggests that the addition of sorafenib after treatments with more intense necrosisinducing effects like TACE may not be effective in suppressing the tumor growth after TACE. The short duration of sorafenib administration (17.1 weeks) can be considered the biggest factor in the failure of this study as well. 
Kudo: Proposal of Endpoints for TACE Combination Trials with Systemic Therapy

\section{BRISK-TA and ORIENTAL Trials}

These two trials did not investigate sorafenib, but two other molecular targeted agents, brivanib and orantinib. Therefore, the trials failed for different reasons $[8,10]$. These reasons will not be discussed in this paper but are discussed elsewhere [16].

\section{Lessons Learned from Negative Trials about Primary Endpoints in the TACE Combination Trials with Systemic Therapy}

OS is generally used as the primary endpoint in Phase III studies of cancer treatments and is also recommended as an endpoint for TACE $[17,18]$. However, the median OS in clinical trials of TACE is never shorter than 18 months, and can even be as long as 32 months, which is a very long time to conduct a clinical trial. In addition, various treatments can be performed as post-trial treatments in patients that withdraw from the trial because of tumor progression or adverse events. The currently approved or available molecular targeted agents include lenvatinib and sorafenib as first-line drugs, and regorafenib, cabozantinib, and ramucirumab as second-line drugs. Patients in either trial arm can also receive post-trial treatment with an immune checkpoint inhibitor (nivolumab). Therefore, it is currently nearly impossible to use OS as an endpoint in clinical trials of combination systemic therapy with TACE in patients with intermediate-stage HCC.

TTP/PFS is sometimes used instead of OS, although it is not always appropriate as a primary endpoint in trials of TACE. There are no data showing that TTP/PFS is a suitable surrogate endpoint for OS. TTP/PFS is usually defined as the time from randomization or treatment initiation to progression as per RECIST or modified RECIST criteria or death; however, it is common practice to perform repeated TACE for original tumor growth or new intrahepatic lesions. In addition, because patients who undergo TACE often have multiple tumors, the development of new intrahepatic lesions is part of the natural course of HCC, and should not be classified as treatment failure (i.e., PD).

Nevertheless, TTP or PFS was the primary endpoint in past trials of combination therapy with sorafenib and TACE, namely, the Phase III Post-TACE trial, the Phase II SPACE trial, and the Phase III TACE-2 trial. Because trials used the RECIST criteria or modified RECIST criteria for response assessment, new intrahepatic lesions had to be classified as PD, naturally ending the protocol treatment. This ultimately reduced the duration of sorafenib administration. The success of the TACTICS trial can be attributed to alterations to the trial design based on lessons learned from these past negative trials, as well as the establishment of a new TACEspecific definition of progression consistent with current TACE use in clinical practice. The TACTICS trial is planning to evaluate OS benefit as a co-primary endpoint when protocolspecified OS events are obtained. If the trial demonstrates an OS benefit, it will provide clear evidence supporting the use of our proposed TTUP-based "TACE-specific PFS" instead of OS as the endpoint for the registration trial for adding a new indication.

We also proposed time to TACE progression (TTTP) as a new measure of TTP appropriate for TACE [19]. PD is defined as a $\geq 20 \%$ increase in the summed diameter of the five largest tumors on post-treatment images relative to baseline images (defined as images from 1 month after treatment) or the detection of extrahepatic spread or vascular invasion, and TTTP is the time from the date of treatment to the date of PD. TTTP is a more clinically appropriate evaluation method for TACE, in addition to correlating well with OS in our validation analysis. As such, we believe that TTTP could be a good surrogate for OS as a primary endpoint in clinical trials of TACE. Izumoto et al. [20] confirmed that TTTP is indeed a good surrogate for OS in trials of TACE. 
Kudo: Proposal of Endpoints for TACE Combination Trials with Systemic Therapy

\section{Conclusion}

Many clinical trials to date have investigated combination therapy with TACE and a molecular targeted agent, and all failed to show improvement of OS and even improvement of TTP or PFS. The TACTICS trial emerged after these failures as the world's first positive study showing improvement of PFS and delay of progression to advanced-stage disease. Its success is attributable to the excellent trial design based on lessons learned from past failed trials of combination therapy with TACE and molecular targeted agents. Both the TACEspecific PFS measure we proposed in the TACTICS trial and TTTP are suitable surrogate endpoints for OS. Therefore, either TACE-specific PFS or TTTP may be a useful surrogate endpoint to OS even in registration trials by pharmaceutical companies which combine systemic therapy $[21,22]$ and TACE.

\section{Disclosure Statement:}

Masatoshi Kudo received lecture fees from Bayer, Eisai, MSD, and Ajinomoto, research grants from Chugai, Otsuka, Takeda, Taiho, Sumitomo Dainippon, Daiichi Sankyo, MSD, Eisai, Bayer, AbbVie, Medico's Hirata, Astellas Pharma, and Bristol-Myers Squibb, and advisory consulting fees from Kowa, MSD, BristolMyers Squibb, Bayer, Chugai, Taiho, Eisai, and Ono Pharmaceutical.

\section{References}

1 Llovet JM, Ricci S, Mazzaferro V, Hilgard P, Gane E, Blanc JF, et al.; SHARP Investigators Study Group. Sorafenib in advanced hepatocellular carcinoma. N Engl J Med. 2008 Jul;359(4):378-90.

2 Cheng AL, Kang YK, Chen Z, Tsao CJ, Qin S, Kim JS, et al. Efficacy and safety of sorafenib in patients in the AsiaPacific region with advanced hepatocellular carcinoma: a phase III randomised, double-blind, placebocontrolled trial. Lancet Oncol. 2009 Jan;10(1):25-34.

3 Li X, Feng GS, Zheng CS, Zhuo CK, Liu X. Expression of plasma vascular endothelial growth factor in patients with hepatocellular carcinoma and effect of transcatheter arterial chemoembolization therapy on plasma vascular endothelial growth factor level. World J Gastroenterol. 2004 Oct;10(19):2878-82.

4 Carmeliet P, Jain RK. Angiogenesis in cancer and other diseases. Nature. 2000 Sep;407(6801):249-57.

5 Wang B, Xu H, Gao ZQ, Ning HF, Sun YQ, Cao GW. Increased expression of vascular endothelial growth factor in hepatocellular carcinoma after transcatheter arterial chemoembolization. Acta Radiol. 2008 Jun;49(5):523-9.

6 Hiraoka A, Kumada T, Kudo M, Hirooka M, Koizumi Y, Hiasa Y, et al.; Real-life Practice Experts for HCC (RELPEC) Study Group and HCC 48 Group (hepatocellular carcinoma experts from 48 clinics). Hepatic Function during Repeated TACE Procedures and Prognosis after Introducing Sorafenib in Patients with Unresectable Hepatocellular Carcinoma: multicenter Analysis. Dig Dis. 2017;35(6):602-10.

7 Kudo M, Imanaka K, Chida N, Nakachi K, Tak WY, Takayama T, et al. Phase III study of sorafenib after transarterial chemoembolisation in Japanese and Korean patients with unresectable hepatocellular carcinoma. Eur J Cancer. 2011 Sep;47(14):2117-27.

8 Kudo M, Han G, Finn RS, Poon RT, Blanc JF, Yan L, et al. Brivanib as adjuvant therapy to transarterial chemoembolization in patients with hepatocellular carcinoma: A randomized phase III trial. Hepatology. 2014 Nov; 60(5):1697-707.

9 Lencioni R, Llovet JM, Han G, Tak WY, Yang J, Guglielmi A, et al. Sorafenib or placebo plus TACE with doxorubicin-eluting beads for intermediate stage HCC: the SPACE trial. J Hepatol. 2016 May;64(5):1090-8.

10 Kudo M, Cheng AL, Park JW, Park JH, Liang PC, Hidaka H, et al. Orantinib versus placebo combined with transcatheter arterial chemoembolisation in patients with unresectable hepatocellular carcinoma (ORIENTAL): a randomised, double-blind, placebo-controlled, multicentre, phase 3 study. Lancet Gastroenterol Hepatol. 2018 Jan;3(1):37-46.

11 Meyer T, Fox R, Ma YT, Ross PJ, James MW, Sturgess R, et al. Sorafenib in combination with transarterial chemoembolisation in patients with unresectable hepatocellular carcinoma (TACE 2): a randomised placebocontrolled, double-blind, phase 3 trial. Lancet Gastroenterol Hepatol. 2017 Aug;2(8):565-75.

12 Bruix J, Raoul JL, Sherman M, Mazzaferro V, Bolondi L, Craxi A, et al. Efficacy and safety of sorafenib in patients with advanced hepatocellular carcinoma: subanalyses of a phase III trial. J Hepatol. 2012 Oct;57(4):821-9.

13 Kudo M, Matsui O, Izumi N, Kadoya M, Okusaka T, Miyayama S, et al.; Liver Cancer Study Group of Japan. Transarterial chemoembolization failure/refractoriness: JSH-LCSGJ criteria 2014 update. Oncology. 2014;87 Suppl $1: 22-31$. 
14 Kudo M, Ueshima K, Torimura T, Tanabe N, Ikeda M, Aikata H, Izumi N, et al. Randomized, open label, multicenter, phase II trial of transcatheter arterial chemoembolization (TACE) therapy In combination with sorafenib as compared with TACE alone in patients with hepatocellular carcinoma: TACTICS trial.J Clin Oncol 36 (suppl; abstr 4017), 2018.

15 Bruix J, Takayama T, Mazzaferro V, Chau GY, Yang J, Kudo M, et al.; STORM investigators. Adjuvant sorafenib for hepatocellular carcinoma after resection or ablation (STORM): a phase 3, randomised, double-blind, placebo-controlled trial. Lancet Oncol. 2015 Oct;16(13):1344-54.

16 Kudo M, Arizumi T. Transarterial Chemoembolization in Combination with a Molecular Targeted Agent: Lessons Learned from Negative Trials (Post-TACE, BRISK-TA, SPACE, ORIENTAL, and TACE-2). Oncology. 2017;93 Suppl 1:127-34.

17 Llovet JM, Di Bisceglie AM, Bruix J, Kramer BS, Lencioni R, Zhu AX, et al.; Panel of Experts in HCC-Design Clinical Trials. Design and endpoints of clinical trials in hepatocellular carcinoma. J Natl Cancer Inst. 2008 May; 100(10):698-711.

18 Llovet JM, Zucman-Rossi J, Pikarsky E, Sangro B, Schwartz M, Sherman M, et al. Hepatocellular carcinoma. Nat Rev Dis Primers. 2016 Apr;2:16018.

19 Arizumi T, Ueshima K, Iwanishi M, Minami T, Chishina H, Kono M, et al. The Overall Survival of Patients with Hepatocellular Carcinoma Correlates with the Newly Defined Time to Progression after Transarterial Chemoembolization. Liver Cancer. 2017 Jun;6(3):227-35.

20 Izumoto H, Hiraoka A, Ishimaru Y, Murakami T, Kitahata S, Ueki H, et al. Validation of Newly Proposed Time to Transarterial Chemoembolization Progression in Intermediate-Stage Hepatocellular Carcinoma Cases. Oncology. 2017;93 Suppl 1:120-6.

21 Kudo M. Immune checkpoint blockade in hepatocellular carcinoma: 2017 update. Liver Cancer. 2016 Nov; 6(1):1-12.

22 Kudo M. Molecular Targeted Agents for Hepatocellular Carcinoma: Current Status and Future Perspectives. Liver Cancer. 2017 Feb;6(2):101-12. 